\title{
Identifying new criteria for assessing the impact on the ecosystem generated by the establishment of Jerusalem artichoke crops on difficult and floodable soils
}

\author{
Florin Nenciu, ${ }^{1, *}$, Gabriel Nae ${ }^{1}$, Gabriela Milian ${ }^{1}$, Iulian Dumitru ${ }^{1}$, Gheorghe Matei ${ }^{2}$, \\ Simona Isticioaia $^{3}$ \\ ${ }^{1}$ INMA Bucharest, Testing Department, Ion Ionescu de la Brad Blvd. No. 6, Bucharest, Romania \\ ${ }^{2}$ University of Craiova, Faculty of Horticulture, Craiova, Dolj, Romania \\ ${ }^{3}$ Agricultural Research-Development Station Secuieni, 377 Principală, Secuieni, Romania
}

\begin{abstract}
Jerusalem Artichoke (Helianthus tuberosus L.) is a technical plant that manages to adapt very well to unfriendly environments, even in polluted or poor in nutrients soils and may serve in the near future as an important raw material for the food, chemical, and pharmaceutical industries. Furthermore, the plant is growing rapidly, producing large amounts of biomass and offers the possibility to be harvested up to 2 times a year, therefore presents a high potential to be used in the field of biofuel production. The plant's capacity to multiply easily by developing small tubers in the soil, allowing the production of economically advantageous crops, can be considered an advantage for biofuel producers, however this feature might be a drawback for agricultural land owners considering the very invasive behavior. The present paper aim to assess two Jerusalem Artichokes crops established on marginal soils, assessing productivity and the extensive impact on the ecosystem, paying a special attention to plant invasiveness tendencies.
\end{abstract}

\section{Introduction}

The biofuels market has grown rapidly in recent years, due to the global goal of limiting the negative effects produced by the widespread use of fossil fuels. In order to meet the increased demand for raw material needed to produce biofuels, very large areas of fertile land have been cultivated with energy plants, creating pressure on the land requirements. Commonly cultivated energy crops are also generating problems associated to the competition with food production, soil depreciation or intensive treatment using various chemicals.

Finding other sources for producing high quality biofuels from new crops, that might grow on marginal, low-grade or contaminated soils, and can develop properly without

${ }^{*}$ Corresponding author: florin nenciu2000@yahoo.com 
intensive agricultural treatments, led us to consider cultivation of Jerusalem artichoke plant. Jerusalem artichoke is a technical plant, that can be used to produce ethanol either from tubers by fermentation and distillation, either from its rich vegetal mass by complex biochemical processes [1-3].

The need to produce large quantities of high-quality biofuels for partially or completely replacement of fossil fuels in global energy production, puts considerable pressure on the use of several plant species with a high potential to be invasive, that can harm either the native species or the ecosystem as a whole [4].

In order to reduce the potential damage, the best solution would be to design easy to use risk assessment systems, capable to evaluate both the positive and negative effects of introducing high-risk energy crops. Although such assessments have been made in the past, their accuracy remains insufficient to produce positive net economic benefits [5]. In addition, risk assessments must be able to assess some non-invasive species that may have negative effects on soils through the economic problems caused by these plants after harvest. The field of risk assessment for potentially dangerous plant species is now in an early stage of development [6], there is a gap of assessment principles and reliable procedures for identifying the invasive potential of plants in various geographic areas. Identifying the potential hazards and opportunities is the first step to build a valuable risk assessment, being needed the design extensive research studies that could assess aspects such as carbon sequestration [7,8], or certain advanced biofuel production techniques [911].

Pest Risk Analysis is commonly defined as a three-stage process (identification, assessing and management of the potential risk) of analyzing biological and other scientific and economic evidence to determine whether a plant should be regulated and the level appropriate measures to be taken [12].

The effort to evaluate and then to manage risks associated with potentially dangerous plant species have to be implemented in a such way to protect human, animal, local plants and soil, without creating unjustifiable obstructions to trading or agriculture. This subject is extremely sensitive, especially if the conclusions of a risk assessment study are taken over by legislative norms that implement various prohibitions.

Plant species that cause serious problems in agriculture are rarely eradicated once they were introduced as crops, and their damages occurs for long periods of time, sometimes imposing aggressive chemical treatment of soils. In order to avoid the costs generated by these management errors and to protect ecosystems in the long term, there is an urgent need to formulate scientific evaluations based on reasonable assumptions.

Most risk studies are performed on whole plant families, either by assuming some probabilities of spread, or by identifying some common hazardous characteristics concentrated within a country or a continent $[13,14]$.

We aim to analyze the characteristics of a single plant, namely Jerusalem artichoke, establishing the crop on a difficult soil (low nutrient level, floodable land, with a high level of compaction, covered with a large number of other persistent weeds). Although the solution to establish a Jerusalem artichoke crop, where other plants have failed to grow in a proper manner (taking into account the plant's ability to adapt to difficult soils), can be beneficial, a number of characteristics regarding the impact on the ecosystem must still be considered. 


\section{Materials and methods}

The presented methodology examines the main elements that can be used to determine how a Jerusalem artichoke crop established on marginal land might pose risks on environment, providing information and guidance to assist farmers in managing the possible hazards generated by this highly adaptive technical plant, based on a range of reasonable assumptions and indicators $[16,17]$.

In the first phase we established what were the main pathways that will allow the plant to spread, and to become a negative issue to the soil, since the identification of highrisk pathways is an important part of an overall weed-risk assessment process.

Historical data on the presence of the plant in the area: We established that Jerusalem artichoke was identified in the history of the area, being eradicated twenty years ago, once the agriculture grow in the area. The plant was not quarantined, however suffered from natural eradication due to the way it multiply, by spreading the tubers.

Pathways to spread: Given that this plant existed in the area and has been eradicated naturally, and additionally it needs agricultural activities (for example plowing), to spread to neighboring lands, for the indicator "Pathways to spread ", Jerusalem artichoke received the low grade for rapid spread on arable land. The fact that it has a well-known dispersal mode that is influenced by anthropic activities and can be easily anticipated, there was another advantage in reducing the level of risk regarding this indicator.

Adaptability on new territories: Another indicator that have been analyzed was the level of adaptability of the plant to new territories. Once entering to new lands, plant species must overcome a range of abiotic and biotic barriers in order to establish. Human activities have an important role in assisting these species to make adaptation easier, because the plants that arrive in small number cannot overcome these barriers.

Accessibility and the cost of intervention on culture: An important role in this case is the accessibility and the cost of intervention on the culture. If in the case of some species of trees introduced for example to stop soil erosion, in the case of turnip the situation is easier.

Monitoring in time: The proper evaluation of plant species becomes relevant when analyzing their behavior through time, in this way the reliability of the prediction will increase.

Ease of eradication: The weed control intensity is actually the difficulty of eliminating an individual plant according to some specific attributes of the plant that makes it persistent. It is calculated as the product between the difficulties of an elimination attempt multiplied by the number of attempts, for a single plant. If in the case of normal soils, this indicator would not have been so important because large tubers can be harvested relatively easily, in the case of difficult soils the tubers usually remain very small and are difficult to harvest.

Reproductive capacity: Species with more than one reproductive system are more invasive, than those with only one system (especially plants that multiply through seeds) Jerusalem artichoke has only one way of propagation, through tubers. Given the particularly high resistance of tubers to drought and unfavorable conditions, we considered this indicator as having a relatively high importance for our evaluation.

Controlling local weeds: The Jerusalem Artichoke plants have proven to be extremely effective in controlling local weeds $(2-5 \%$ of other plants have been identified in the crop), and for marginal agricultural land, this is a key factor. 
Invasive behavior of the plant: Establishment of other crops after Jerusalem Artichoke culture is difficult especially on difficult/marginal soils, because many tubers will be too small to be harvested, and will germinate next year, affecting future crops.

Carbon sequestration: The crop has a rich plant mass even in the case of wild propagation, therefore in the case of integration into the soil it has superior advantages regarding carbon sequestration.

Connections with local animals and birds: Species of birds (pheasants) and animals (rabbits) have been identified in the Jerusalem artichoke culture, the high and dense vegetation representing shelter for them.

\section{Results and discussion}

\section{Establishing and evaluating Jerusalem artichoke crops}

Jerusalem artichoke (Helianthus tuberosus), is an herbaceous perennial plant that has a high productivity of tubers rich in inulin, which is a fructan polymer [15]. These inulins can be broken down into fructose and glucose for conversion into ethanol by fermentation.

We set ourselves the goal of identifying the differences between the characteristics of a Jerusalem artichoke crop established on difficult soil (difficult to process, floodable and deprived of nutrients) and a crop established on a productive land, having optimal planting conditions and works. In the two established plant cultures we evaluated some indicators envisaging mainly the health of the ecosystem. In order to observe the real invasive effect of the Jerusalem artichoke on the difficult terrain, we monitored the crop for three years (in the first year the tubers were planted and harvested, while in in the next two years we let the crop grow in the wild environment, the single work done being plowing the land in autumn).

A planting installation designed and executed by INMA Bucharest (Figure 1) was used for planting the tubers, towed by an agricultural tractor TD 80D New Holland 65 CP. Have been planted tubers without mechanical damages (having length between 3-10 $\mathrm{cm}$ ), the machine being adjusted to plant at $100 \mathrm{~cm}$ distance per row, at $9 \mathrm{~cm}$ depth, the distance between the rows being $80 \mathrm{~cm}$ The working speed of the equipment was set to $1.5 \div 1.9 \mathrm{~km} / \mathrm{h}$, being equipped with a water spraying installation incorporated in the system.

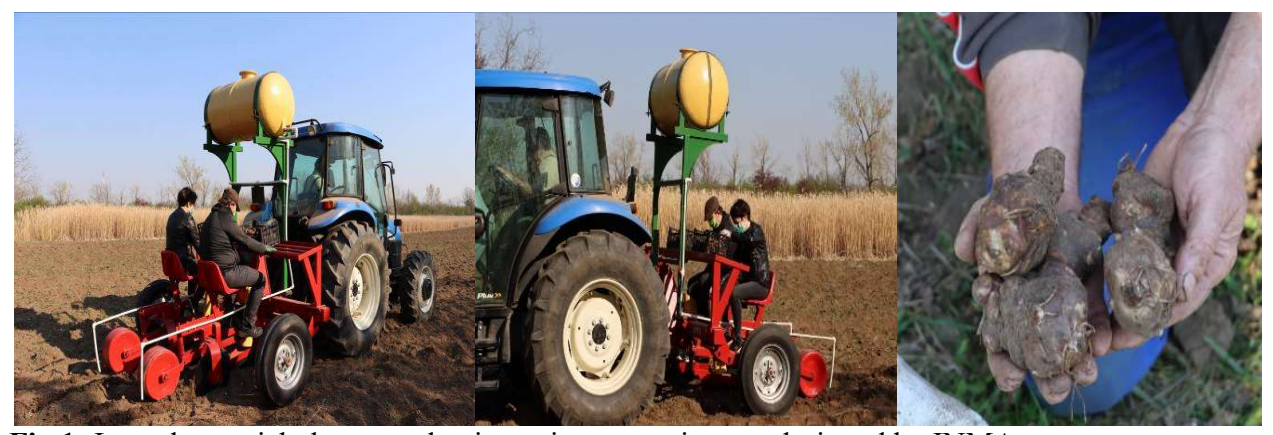

Fig.1. Jerusalem artichoke crop planting using an equipment designed by INMA.

Measurement and calculation of the main indicators in order to assess the impact on the ecosystem

In addition to the potential for ethanol production on the two crops, we aimed to analyze the effect that wild-grown culture has on the ecosystem. Unlike classical 
invasiveness studies, we also considered the positive elements that this plant can have on the ecosystem when it is planted on difficult agricultural soils. Figure 2 is suggestive for the plant grown wild from the bulbs remained in the soil, in the $3 \mathrm{rd}$ year after planting. Can be seen that Jerusalem artichoke plant growth is not much affected by the e soil type even if it is low in nutrients, managing to adapt and thrive in difficult soils.

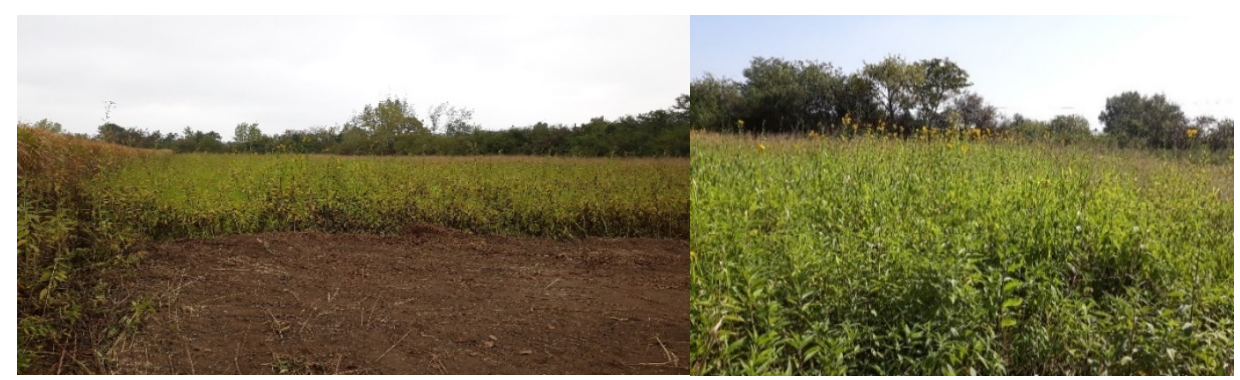

Fig. 2. Jerusalem artichoke crop grown wild from the tubers that remained in the soil, in the third year after planting

Although the vegetal mass was abundant, have been noticed that productivity on difficult soil was much lower, the tubers remaining very small and undeveloped.

The main elements that contributed to the underdevelopment of the tubers were the lack of precipitation (the crop is not irrigated), the high level of soil compaction and the high density of plants grown per square meter.

Figure 3 exemplifies the measurements made to evaluate the culture parameters, such as the number of plants grown per square meter, plant sizes, the number of tubers harvested per square meter, the number of inflorescences and plant vegetal mass.

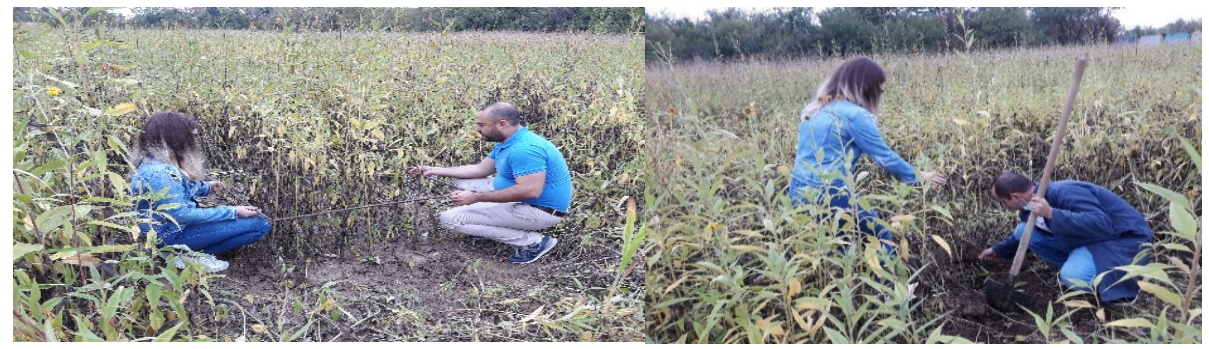

Fig. 3. Evaluating the development parameters of a Jerusalem artichoke crop grown wild on difficult soils

The benefits of the crop such as high nectar production, rich plant mass that allows carbon sequestration in the soil, the potential to represent a habitat for birds and animals [18], were all assessed using techniques based on indicators and scoring. (Fig. 4).

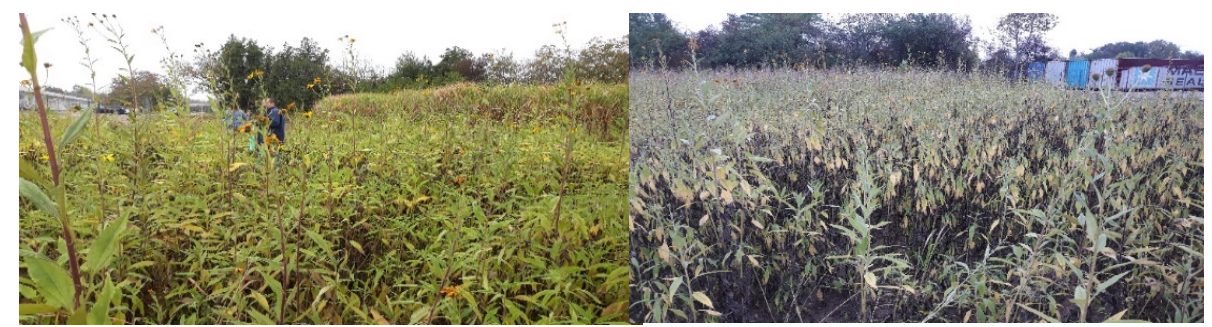


Fig. 4. Highlighting the honey potential of Jerusalem artichoke culture in different time periods

We also evaluated the potential of Jerusalem artichoke crop to control local weeds on difficult soils, considering that this plant might impose when competing with very persistent plants. In the case of wild-grown crops, Jerusalem artichoke have proven to be very effective in controlling local weeds, leaving only a $1-4 \%$ percentage of other plants to grow, and even these have been found underdeveloped.

The weeds that could still be identified in the Jerusalem artichoke culture were Amaranthus retroflexus, Setaria viridis, Carduus nutans, Senecio Vulgaris, but in a very small percentage (Figure 5).

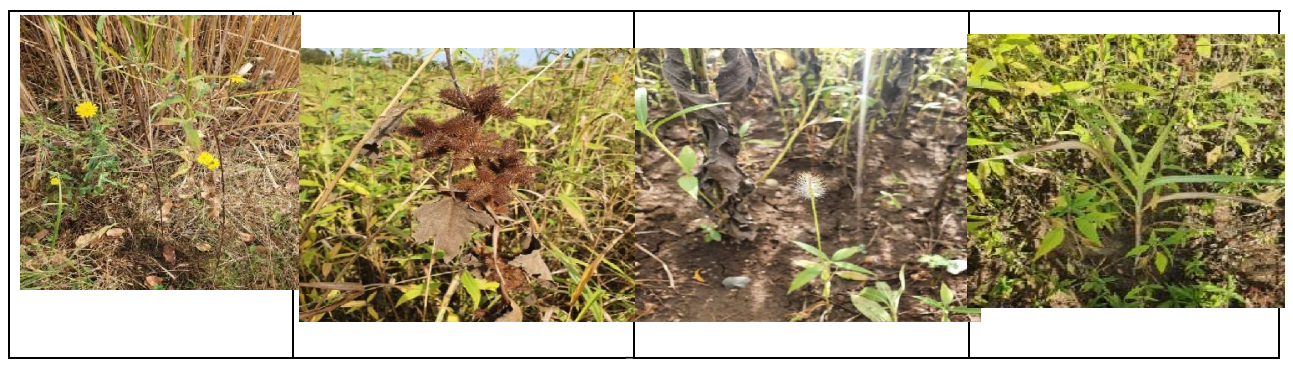

Fig. 5. Jerusalem artichoke control of local weeds on difficult soils

Productivity for the crops established on soils with different characteristics (fertile soils and optimal conditions versus difficult soils and unfavorable conditions), showed a much lower productivity of the total quantity of tubers produced on wild-grown crops. Analyzing 1 square meter from twenty plots, collected from both cultures, were found differences up to $40 \%$ productivity in favor of fertile lands (Figure 6 ).

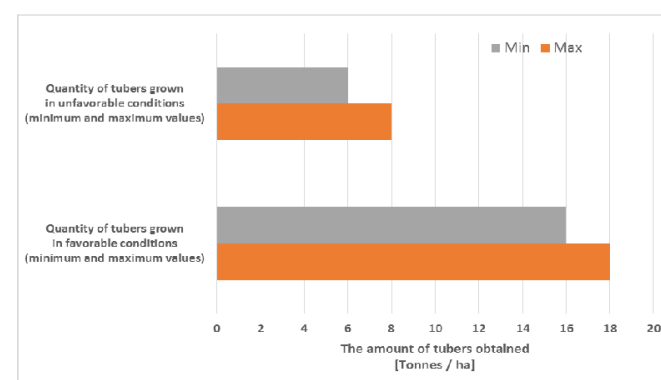

Fig. 6. Comparative analysis of the mass of tubers produced both in favorable planting conditions and in unfavorable planting conditions (minimums and maximums recorded for each category)

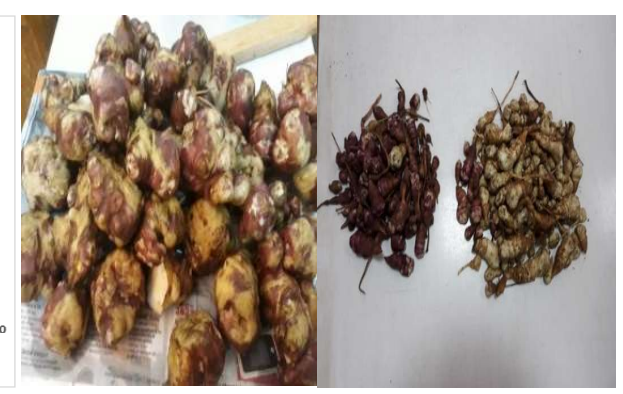

Fig. 7. Differences between the dimensions of the tubers obtained in the two cultures favorable planting conditions first picture) and in unfavorable planting conditions (second picture)

Not only the number but also the size of the tubers was affected, being smaller by up to 10 times, as can be seen in figure 7 . The size of the tubers was actually the element that made the difference. The size of the tubers was actually the element that made the difference in the productivity for the two cultures. A determining factor here is the amount of rainfall, which influenced decisively the wild-grown crop, that has not been irrigated.

The biomass production of the two crops have been comparatively assessed in order to associate with carbon sequestration and with the potential to produce ethanol from conversion of the stems to alcohols, using thermochemical process. By incorporating the 
vegetal mass in the furrow, can also obtain an improvement of the soil quality [18]. In the thicket formed by the Jerusalem artichoke stems, different types of birds and animals can find shelter against predators and can nest, this being a very valuable element for the ecosystem. There have been determined low variations in the amount of stems mass per hectare (Figure 8), the wild-grown crop developing larger number of plants while the fertile soils allowed the development of taller and thicker stems, but fewer in number.

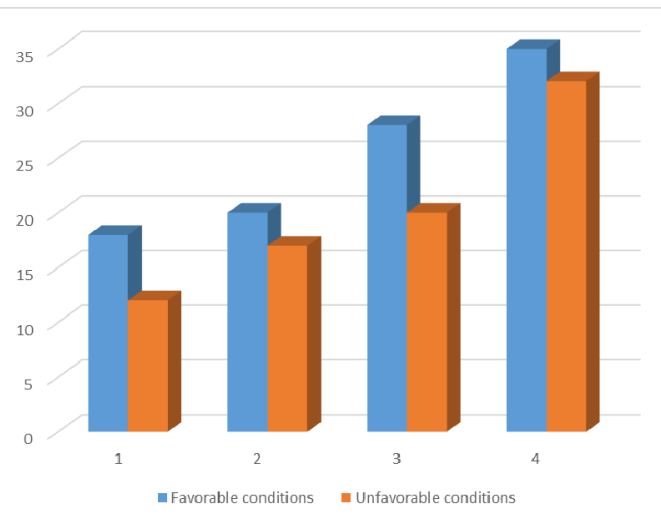

Fig. 8. Comparative analysis between plant mass of stems for favorable conditions versus unfavorable conditions

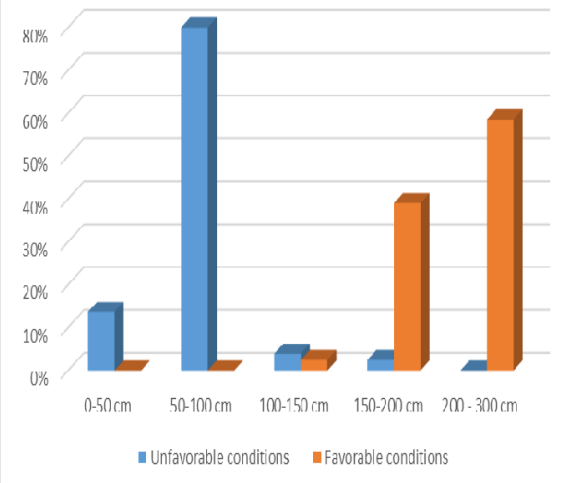

Fig. 9. Plant height analysis for Jerusalem artichoke crops

Numerous differences have been identified regarding the plants size assessment on the two cultures. Plants grown on fertile land were much taller, a large share being in the height ranges between 150-200 cm and 200-300 cm. In the case of crops established on difficult land, the vast majority of plants were in the range of $50-100 \mathrm{~cm}$, but they were in a much larger number. Among the causes that generated the difference, were on the one hand the drought (the crop established on the difficult soil has not being irrigated) and the fact that on the fertile land the planting technology was established with the standardized distances between rows, while on the wild culture, the plants grew chaotically (Figure 9).

In terms of alcohol production, Jerusalem artichoke has a lower potential than other similar plants (such as potatoes), due to the difficulties in converting inulin to sugars. From a qualitative point of view, the differences between the tubers harvested from the two soil types was not different, although from a quantitative point of view, the differences in tonnage of tubers per hectare were large.

Although the main indicators are presented in the following table according to the impact on the ecosystem, the results have been assessed chronologically on three stages: Planting stage (ease of planting, planting mechanization, planting efficiency), Plant development and monitoring (maintenance works, effect on weed eradication, plant adaptability, easy monitoring, pest resistant, etc.) and Harvesting stage (tuber productivity, vegetal mass productivity, easy eradication etc.). 
Table 1 Centralizing table of the main indicators for assessing the impact on the ecosystem, following the establishment of a Jerusalem artichoke crop on difficult soils.

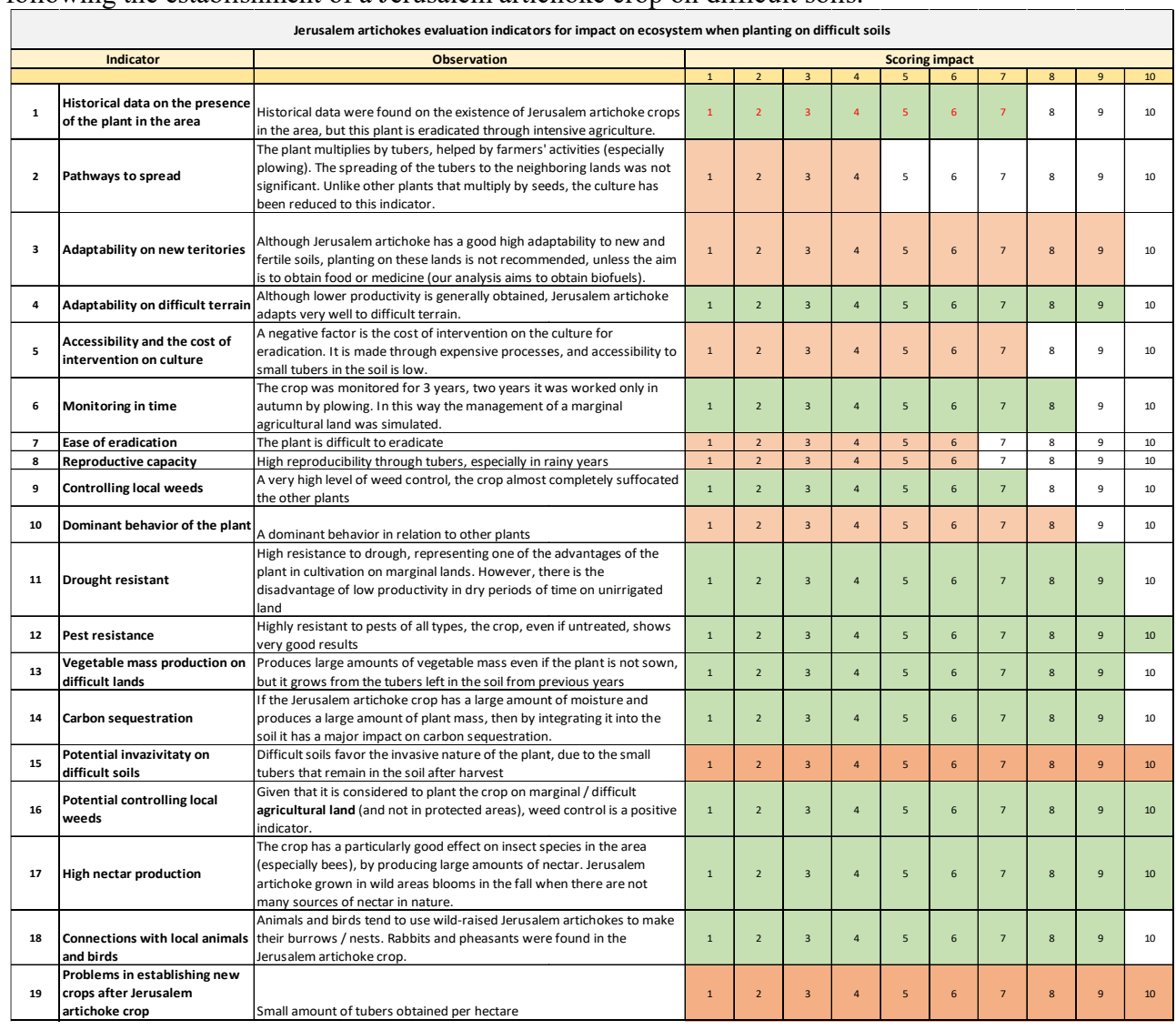

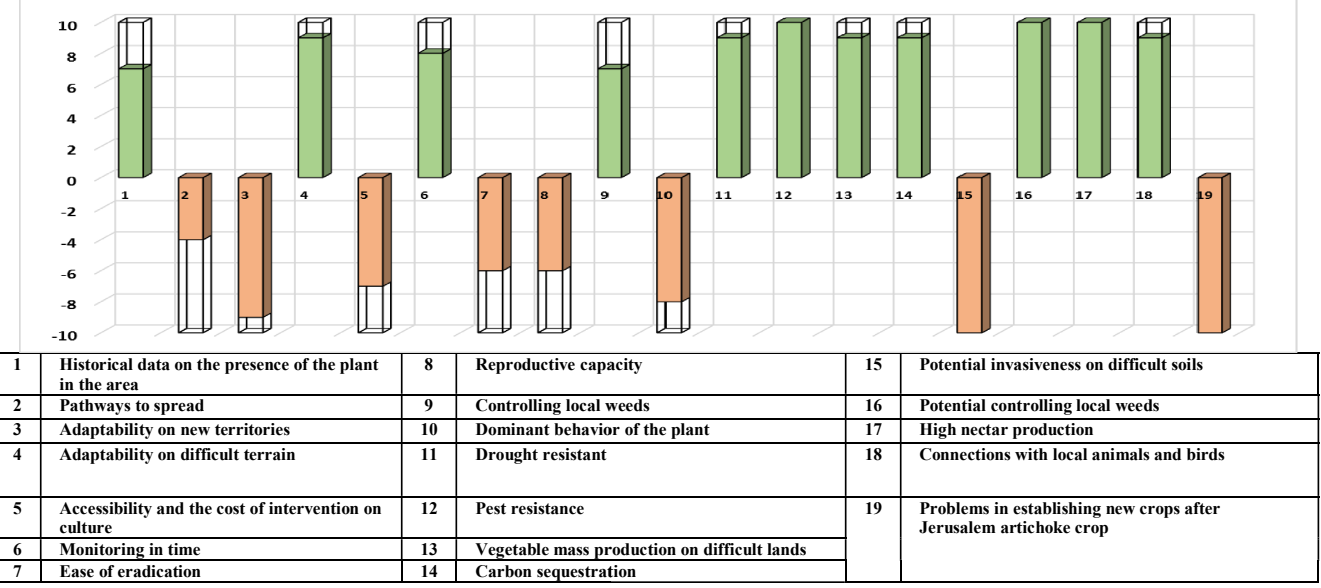

Fig. 10. Graphic representation of the influence of a Jerusalem artichoke crop established on difficult soils on the ecosystem, where the indicators 1-19 are listed above 


\section{Conclusions}

In order to ensure the reduction of our dependence on fossil fuels, it is mandatory to develop the domain of biofuels, both for those obtained from planting technical crops marginal or depleted soils and biofuels obtained from biomass waste.

We have highlighted in this article that Jerusalem artichokes crop have a high potential for ethanol production, and due its special properties of adaptability and resistance to extreme drought conditions, can be planted on marginal, polluted, nutrientfree or floodable areas.

By analyzing the problems generated by this crop in terms of invasiveness and the effects generated on the ecosystem, farmers can decide consciously whether Jerusalem artichoke present a high risk or is a good opportunity.

Jerusalem artichokes crops showed a good growth in difficult conditions and in less fertile soils, even though the humidity have been regulated only by rain. Without irrigation and agricultural work, the culture succeeded to grow a vegetable mass in the less fertile land was maintained at values close to the values obtained on fertile lands.

However, the quantity of tubers produced, suitable for producing alcohol was very small in the case of crops on difficult lands, in the best cases being $40 \%$ smaller than those in ordinary lands.

Jerusalem artichokes shoed a very good control of local weeds, only $1-4 \%$ of other plants survived in a culture dominated by Jerusalem artichoke for a period of 3 years.

Planting a crop on marginal lands does not necessarily affect other planned crops. However, in the case of fertile lands, if the farmer desired to establish another crop after Jerusalem artichoke, he must consider planting a very resistant variety to compete with the tubers remained in the soil.

In terms of impact on the ecosystem, Jerusalem artichokes crops offers important benefits, especially for wild-grown plants. It is a shelter for some species of animals and plants, and is a honey producing plant very appreciated by insects because it produces nectar in late autumn periods.

Physico-chemical analyzes showed similar potential for alcohol production in the two crops, however on the culture established on difficult soils a much smaller quantity of tubers was obtained. Because there are many variables in the analysis of production from a quantitative point of view, such as the presence of precipitation (since in the analyzed year the average annual amount of precipitation in the area was very low), for a better estimation a multiannual analysis is needed.

This work was supported by a grant of the Romanian Ministery of Research and Innovation CCDI UEFISCDI, project number PN-III-P1-1.2-PCCDI-2017-0566, Contract no. 9PCCDI/2018, and PNCDI III, contract no. 16PFE

\section{References}

1. A.Eliasson, Master thesis, Lund University, Department of chemical Engineering, Sweden, (2017)

2. S.J.Kays, S.F., CRC Press Taylor \& Francis Group, New York, United States, (2008). 
3. Y. Qiu, P. Lei, Y. Zhang, Y. Sha, Y. Zhan, Z. Xu, S. Li, H. Xu, P. Ouayang, Biotechnology for biofuels, 11, 151, (2018)

4. R. Meyer, A. Grunwald, C. Rösch, A. Sauter, Working report, 121, Germany, (2007).

5. Y. Wu, F. Zhao, L. Wang, L. Qiu, G. Alexandrov, Geoscience Letters, 5 (14), (2018)

6. A. Bielecki, S. Ernst, W. Skrodzka, I.Wojnicki, Environmental Science and Pollution Research, 27, 11506-11530, (2020)

7. E.C. Toochi, Forestry Research and Engineering : International Journal, 2 (3), Nigeria, (2018)

8. B. Zheng, D. TongLi, M. Liu, F. Hong, C. Geng, G. Li, H. Li X, L. Peng, J. Qi, L. Yan, Y. Zhang, H. Zhao, Y. Zheng, K. He, Q. Zhang, Atmos. Chem. Phys., Trends in China's anthropogenic emissions, 18, 14095-14111, (2018)

9. S.S. Singh, R. Dhaliwal, M. Puri, Society for Industrial Microbiology, 35, 777-782, (2008)

10. P. Thanokeo, S. Thanonkeo, K.Charoensuk, M. Yamada, African Journal of Biotechnology, Research Gate, 10(52), 10612-10617, (2011)

11. V. Starovoytov, O. Starovoytova, N. Aldoshin, A. Manohina, Acta Technologica Agriculturae, 1, 7-10, Slovacia, (2017)

12. European Environment Agency, EEA Technical report No 4/2008, 4, ISSN 1715 2237, (2008)

13. J. Popp, Z. Lakner, M. Harangi-Rákos, M. Fári, Renewable and Sustainable Energy Reviews, Elsevier, 559-578, (2014)

14. A. Hina Fathima, K. Priya, T. Sudakar Babu, K.R. Devabalaji, M. Rekha, K. Rajalakshmi, C. Shilaja, Ijiret, 3 (1), (2014)

15. S. Bhagia, H. Akinosho, J.F.S. Ferreira, Biofuel Research Journal, 14, 587-599, (2017)

16. E. Aguaron, E.G. McPherson, Urban Ecosystems and Social Dynamics Program, 4371, USA, (2012)

17. U.S. Environmenal Protection Agency, Combined Heat and Power Partnership, USA, (2015)

18. F. Nenciu, V. Vlăduț, IOP Conf. Ser.: Earth Environ. Sci., 635, 1-13, (2021) 